\title{
Research Article \\ Histopathology of Cutaneous Leishmaniasis Caused by Leishmania donovani in Sri Lanka
}

\author{
Harshima Wijesinghe $\mathbb{D}^{\mathbb{D}},{ }^{1}$ Nayana Gunathilaka $\mathbb{D}^{2},{ }^{2}$ Saveen Semege, ${ }^{3}$ Nishantha Pathirana, ${ }^{4}$ \\ Nuwani Manamperi, ${ }^{2}$ Chandu de Silva $(1),{ }^{1}$ and Deepika Fernando ${ }^{5}$ \\ ${ }^{1}$ Department of Pathology, Faculty of Medicine, University of Colombo, Sri Lanka \\ ${ }^{2}$ Department of Parasitology, Faculty of Medicine, University of Kelaniya, Sri Lanka \\ ${ }^{3}$ Army Preventive Medicine \& Mental Health Services, Army Headquarters, Sri Jayawardenepura, Sri Lanka \\ ${ }^{4}$ Army Hospital, Colombo, Sri Lanka \\ ${ }^{5}$ Department of Parasitology, Faculty of Medicine, University of Colombo, Sri Lanka
}

Correspondence should be addressed to Harshima Wijesinghe; harshima@path.cmb.ac.lk

Received 13 January 2020; Revised 31 March 2020; Accepted 13 April 2020; Published 5 May 2020

Academic Editor: Rosario Caltabiano

Copyright (c) 2020 Harshima Wijesinghe et al. This is an open access article distributed under the Creative Commons Attribution License, which permits unrestricted use, distribution, and reproduction in any medium, provided the original work is properly cited.

\begin{abstract}
Cutaneous leishmaniasis (CL) is a neglected tropical disease that is gaining importance in Sri Lanka and internationally. The clinical presentation, pathology, and method of parasite elimination in CL vary according to the species. Leishmania donovani is the causative organism for leishmaniasis in Sri Lanka. This collaborative cross-sectional study describes the clinicopathological features of cutaneous leishmaniasis among personnel of the tri-forces serving in the North and East of the country. The histology of fifty cases of CL confirmed by at least two methods (slit skin smear, lesion aspirate, tissue impression, and histology) was reviewed. The parasitic load was assessed semiquantitatively. The histological features were correlated with the clinical presentation and organism load. The majority $(89.8 \% ; n=44)$ presented with a single lesion mostly located in the upper limb (69.4\%). The lesion types included papule (34.7\%), nodule (32.7\%), and an ulcer (30.6\%). The evolution time of lesions averaged 31.55 weeks. Epidermal changes were observed in 49 of the biopsies and included hyperkeratosis $(90.0 \%$; $n=45)$, acanthosis $(44.0 \% ; n=22)$, atrophy $(34.0 \% ; n=17)$, and interface change $(66 \% ; n=33)$. Dermal changes were seen in all cases and were characterized by a lymphohistioplasmacytic inflammatory infiltrate of variable intensity with ill-formed granuloma in 19 cases (38\%) and well-formed epithelioid granulomas in 22 cases (44\%). Focal necrosis was present in 20\% $(n=10)$. Leishmania amastigote forms were observed in $88 \%(n=44)$. Transepidermal elimination $(P=0.025)$, granuloma $(P=0.027)$ formation, and type of lesion $(P=0.034)$ were significantly associated with the organism load. Granuloma formation was associated with a reduction in organism load, indicating that the macrophage activation played an important role in the control of the organism.
\end{abstract}

\section{Background}

Leishmaniasis, a neglected tropical disease, is caused by the intracellular protozoan of the genus Leishmania. Of the three main forms of clinical presentations seen with leishmaniasis, cutaneous leishmaniasis (CL) is the most common with an estimated 600,000 to one million new cases occurring worldwide annually [1]. The estimated global burden of this disease is believed to be higher than the reported numbers due to underreporting, under surveillance, and inadequate case detection techniques [2]. Since 1993, the geographical distribution of leishmaniasis is reported to have expanded significantly in the world, with a concomitant sharp increase in the number of cases [3] and emerging new disease foci [4]. Several countries have experienced epidemics including South Asian countries of Nepal, Bangladesh, and India $[5,6]$. The first locally acquired case of CL was reported in Sri Lanka in 1992 [7], and it is currently an established parasitic disease in the country. Leishmania donovani MON-37 has been reported to be the causative organism of both cutaneous 
and visceral leishmaniasis in Sri Lanka $[8,9]$. CL presents a spectrum of clinical and histopathological manifestations that encompass different morphological states such as nodule, plaque, and ulcer, and which may persist as a chronic lesion or heal with a scar, depending on the infecting parasite species [10]. The histopathological spectrum of changes seen in cutaneous leishmaniasis ranges from a diffuse infiltrate of macrophages, lymphocytes, and plasma cells to ill-defined granuloma to granulomatous inflammation with welldefined granuloma [11]. A single study conducted in Sri Lanka based on 31 skin biopsies described that lesions of CL could be classified into four histological groups I-IV that were similar to that of the spectrum seen in leprosy ranging from lepromatous to tuberculoid leprosy. The histological groups from I-IV showed a significant inverse relationship with the mean parasitic index [12]. Studies conducted elsewhere too have shown that organism load decreases progressively with the development of granulomatous inflammation [11] indicating that the development of granuloma is associated with increased control of the organism. Elimination of the parasite in cutaneous leishmaniasis has been thought to involve two mechanisms, activation of intact macrophages and a necrotizing process that destroys the macrophage with the parasite it contains [13-17]. Most studies have shown the latter to be the more efficient mechanism for parasite elimination $[14,18,19]$. However, a study conducted in Sri Lanka showed that necrosis was not a prominent feature and the main mode of parasite elimination was thought to be macrophage activation giving rise to epithelioid granulomata [12]. Currently, there are about 20 species of Leishmania that may cause CL. The clinical presentations, pathology, and method of parasite elimination vary according to the species. Therefore, understanding the immune mechanisms required for the control of infection within the varied tissue microenvironments that contain Leishmania is a key factor for developing effective vaccines and therapeutics. Evaluation of the histopathological features in a Sri Lankan population would facilitate a better understanding of host-parasite interaction. There is currently only one published study on the histopathology of CL due to $L$. donovani which is the causative organism of leishmaniasis in Sri Lanka [12]. Hence, the present study is aimed at describing the histopathological features of skin biopsies from patients with CL in Sri Lanka and correlating the histopathological characteristics with the organism load and clinical presentation.

\section{Methods}

2.1. Study Setting and Design. This was a collaborative crosssectional descriptive study conducted by the Departments of Pathology and Parasitology of the Faculties of Medicine of the Universities of Colombo and Kelaniya and the Sri Lanka Army Medical Corps from November 2018 to November 2019. Clinical material collected from skin lesions of personnel of the tri-forces serving in the North and East of the country with features suspicious for CL was evaluated for the presence of Leishmania organisms by slit skin smear, lesion aspirate, tissue impression, and histology. All cases in which leishmania organisms were detected by at least two of the methods (slit skin smear, lesion aspirate, tissue impression, and histology) were included. Cases in which tissue was unsuitable for histological analysis due to severe crushing or autolysis were excluded. Ethical clearance for the study was obtained from the Ethical Review Committee of the Faculty of Medicine, University of Kelaniya, Ragama, Sri Lanka (P/94/02/2019). Permission to conduct the study was also obtained for the administration of the Sri Lanka Army.

2.2. Sample Collection. Two punch biopsies, each measuring 2-3 mm, were obtained from the lesion under local anaesthesia under aseptic conditions. An impression smear was obtained from the one biopsy which was thereafter fixed in $10 \%$ formalin for routine histopathological processing. Impression smears made on glass slides were air-dried, fixed in methanol, stained with Giemsa, and examined under a light microscope for the presence of parasites.

Slit skin smears were prepared by making a nick at the active edge of the lesion. Material from the gaped walls of the nick was scraped using the blunt side of the scalpel blade, and a thin skin smear was prepared. If the lesion had a scab, it was removed, and tissue material was scraped from the base of the lesion [4] to prepare the smear. Smears from fineneedle aspirates were obtained by injecting $0.1-0.2 \mathrm{ml}$ sterile saline into the edge of the lesion with a $23 \mathrm{G}$ needle fixed to a $2 \mathrm{ml}$ syringe and applying suction while performing rotator to and fro movement. The slides prepared from skin scrapes and FNA were air-dried, fixed in methanol, followed by staining with Giemsa, and examined under oil emersion using a stereomicroscope for the presence of oval-shaped amastigotes with the characteristic dot and dash appearance. The sides were cross-checked by a consultant parasitologist as a quality control procedure.

2.3. Evaluation of Light Microscopy. All biopsies from the lesions clinically suspicious of CL were evaluated by two histopathologists at the Department of Pathology, Faculty of Medicine, Colombo. All tissue samples were dehydrated, cleared, embedded in paraffin, cut into $4-5 \mu \mathrm{m}$ thick sections, and stained with Haematoxylin and Eosin. Giemsa stain was performed in cases where the organisms were not detected on Haematoxylin and Eosin. The sections were assessed for histological features found in the epidermis and the dermis. The epidermis was evaluated for the presence of ulceration, crusting, transepidermal elimination, intraepidermal neutrophils, hyperkeratosis, parakeratosis, acanthosis, atrophy, spongiosis, exocytosis, and interface change. The dermis was evaluated for the intensity of inflammatory infiltrate, as well as the presence of different cell types in the infiltrate, the formation of granuloma, and the parasite load.

The inflammatory infiltrate in the dermis was categorized as mild, moderate, and extensive. A semiquantitative analysis of the constituting inflammatory cells (lymphocytes, macrophages, plasma cells, neutrophils, and eosinophils) was performed.

Based on the semiquantitative assessment, the infiltrate was categorized as macrophage predominant, lymphocyte predominant, predominantly lymphohistiocytic, 
and predominantly lymphoplasmacytic and having equal numbers of lymphocytes, plasma cells, and histiocytes.

The presence of necrosis was categorized as diffuse, focal or individual cell necrosis, and absent. Multinucleated giant cells, vasculitis, and neuritis were documented as being present or absent. The presence of granulomas was documented as absent, ill-defined, and well-defined. The organisms were quantified based on the Ridley parasitic index [15], and the overall histological pattern was categorized according to the modified Ridley's criteria [16]. The histological features were correlated with the nature of the lesion (patch, nodule, and plaque), the duration of the lesion, and organism load. The data analysis was conducted using the SPSS software package (version 23).

\section{Results}

Seventy-eight soldiers underwent further investigation for cutaneous lesions suspicious of leishmaniasis. The CL organisms were detected by at least one of the methods used in 63 cases $(80.7 \%)$. Ten cases were positive only on histology, one only on slit skin smear, and one only on lesion aspirate. Therefore, these 12 cases were excluded. One case was excluded because the tissue was poorly preserved and not suitable for further histological evaluation. The remaining 50 cases were included in the study.

3.1. Clinical Features. Of the 50 patients with confirmed CL, only two were female (4\%). Their age ranged from 19 to 54 years with an average age of 32.24 years. The evolution time of lesions varied from 3 to 660 weeks with an average of 31.55 weeks. The majority $(89.8 \% ; n=44)$ presented with a single lesion, while four presented with two lesions and one with three lesions. The lesions were mostly located in the upper limb (69.4\%), followed by back (10.2\%), and lower limbs $(8.2 \%)$. The lesion types included papule $(34.7 \%)$, nodule $(32.7 \%)$, and ulcer $(30.6 \%)$. Only one patient presented with a plaque lesion.

3.2. Pathological Features: Epidermal Changes. The histopathological analysis from the 50 skin biopsies of patients with clinical and laboratory of localized CL showed morphological alterations in both, epidermis and dermis. Alterations of the epidermis were observed in 49 skin biopsies. Hyperkeratosis was present in $45(90.0 \%)$ of the biopsies with it being the only epidermal change seen in five cases $(10.0 \%)$. Acanthosis was present in $22(44.0 \%)$ cases, while the epidermis was atrophic in $17(34.0 \%)$. The majority $(66 \% ; n=33)$ of biopsies showed interface change with varying degrees of basal cell degeneration and pigmentary incontinence. Other changes included parakeratosis in $42.0 \%(n=21)$, spongiosis and exocytosis in $34.0 \%(n=17)$, and ulceration in $14.0 \%$ $(n=7)$. Seven of the biopsies (14\%) showed transepidermal elimination of amastigotes, while crusting and intraepidermal neutrophils were present in $12.0 \%(n=6))$ and $26 \%$ $(n=13)$, respectively (Table 1$)$.

3.3. Pathological Features: Dermal Changes. Dermal changes were seen in all cases and were characterized mainly by a lymphohistiocytic inflammatory infiltrate of variable inten-
TABLE 1: Epidermal changes of lesions among patients with cutaneous leishmaniasis.

\begin{tabular}{lcc}
\hline Epidermal change & Present & Absent \\
\hline Ulceration & $7(14 \%)$ & $43(36 \%)$ \\
Transepidermal elimination of amastigotes & $7(14 \%)$ & $43(36 \%)$ \\
Hyperkeratosis & $45(90 \%)$ & $5(10 \%)$ \\
Parakeratosis & $21(42 \%)$ & $29(58 \%)$ \\
Acanthosis & $22(44 \%)$ & $28(56 \%)$ \\
Atrophy & $17(34 \%)$ & $33(66 \%)$ \\
Spongiosis & $17(34 \%)$ & $33(66 \%)$ \\
Exocytosis & $17(34 \%)$ & $33(66 \%)$ \\
Interface change & $33(66 \%)$ & $17(34 \%)$ \\
Crusting & $6(12 \%)$ & $44(88 \%)$ \\
Intraepidermal neutrophils & $13(26 \%)$ & $37(74 \%)$ \\
\hline
\end{tabular}

sity (Figure 1(a)). Associated plasma cells were seen in $90 \%$ $(n=45)$. The inflammatory infiltrate was intense in $58 \%$ $(n=29)$, moderate in $32 \%(n=42)$, and mild in $10 \%(n=5)$ of the cases. It was characterized by a predominance of histiocytes in $44 \%$, followed by lymphocytes in $14 \%$, and comprised approximately equal numbers of lymphocytes, plasma cells, and histiocytes in $22 \%(n=11)$. Neutrophils were seen in 34 cases $(68 \%)$, while eosinophils were seen in fewer numbers and less frequently $(14 \% ; n=7)$. Granuloma formation was present in 41 cases with ill-formed granuloma in 19 cases [38\%] (Figure 1(b)) and well-formed epithelioid granulomas in 22 cases (44\%) (Figure 1(c)). Multinucleated giant cells were seen in $26 \%(n=13)$ and focal necrosis area in $20 \%(n=10)$. None of the lesions showed vasculitis, but perineural inflammation was present in $10 \%$ (Table 2 ).

3.4. Detection of the Parasite. Leishmania amastigote forms were observed in $66 \%(n=33)$ of HE-stained histological sections and seen only on Giemsa in 11 (22\%) cases (Figure $1(\mathrm{~d})$ ). The parasite load ranged from 0 to 6 on the Ridley parasitic index (Table 3).

Most of the lesions were of Modified Ridley index (RMI) group $2(38 \% ; n=19)$ or group $3(34 \% ; n=17)$. One case in which histology was negative for organisms showed a perivascular chronic inflammatory infiltrate with no evidence of granuloma, so it did not fit into any of the RMI categories (Table 4). In patients with CL lesions of 24 weeks or less (early lesions), $85.7 \%$ were positive and $14.3 \%$ were negative; and in patients with lesions of more than 24 weeks of infection (late lesions), all seven lesions were positive.

3.5. Correlation of Clinicopathological Features and Organism Load. The clinical presentation (the type of lesion, duration of the lesion), epidermal changes, nature of dermal infiltrate, and presence of granuloma and RMI were correlated with the RPI using chi-squared analysis. Transepidermal elimination $(P=0.025)$, granuloma $(P=0.027)$ formation, and type of lesion $(P=0.034)$ were significantly associated with the organism load. Higher parasitic loads of RPI $=4$ and above showed a positive association with transepidermal elimination and a negative association with well-defined granuloma. 


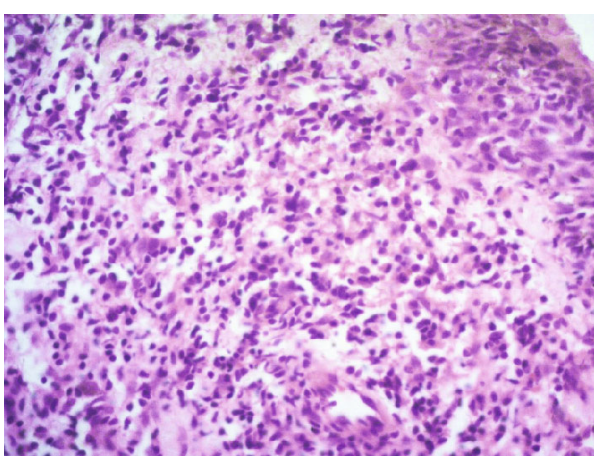

(a)

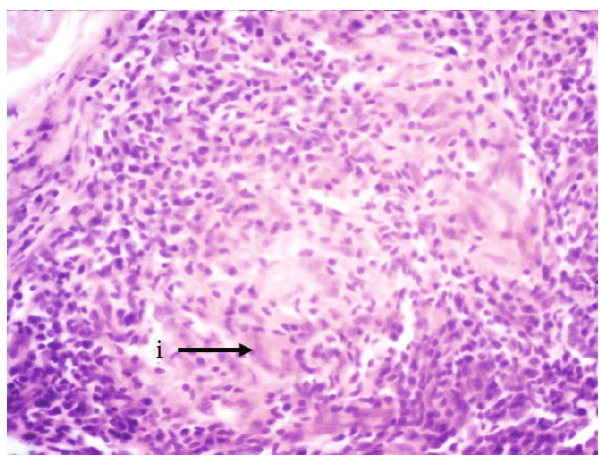

(c)

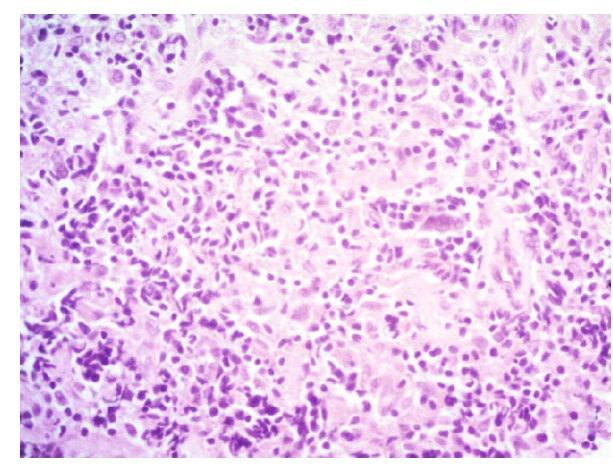

(b)

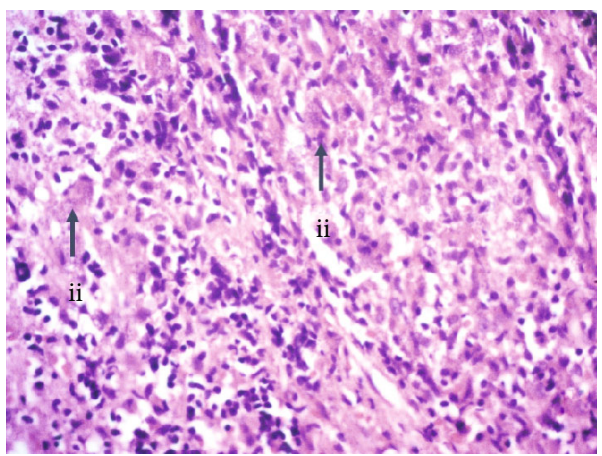

(d)

FIGURE 1: The spectrum of histopathological changes seen in the dermis (haematoxylin and eosin stain $\times 400)$ : (a) diffuse lymphohistioplasmacytic infiltrate, (b) ill-defined granuloma, (c) well-defined granuloma with (i) multinucleated giant cells, and (d) diffuse lymphohistioplasmacytic infiltrate showing (ii) Leishmania amastigotes within macrophages.

All lesions presenting as nodules showed organisms on histology, while the single lesion presenting as a plaque was negative for organisms. Of the biopsies that were negative for organisms on histology, apart from the single lesion that showed nonspecific perivascular inflammation, all other biopsies showed well-defined epithelioid granuloma. None of them showed exocytosis or spongiosis. All were from early lesions ( $<24$ weeks). Lesions with a low RPI $(\mathrm{RPI}=1-3)$ were more likely to be of RMI group 3 than RMI group 1, whereas lesions with a high RPI (RPI $=4$ or above) were more likely to be of RMI group 1 than $3(P<0.001)$.

The 12 cases which were excluded, since organisms were detected only on one of the four detection methods, were reviewed. The two cases that had shown organisms only in slit skin smear and only in tissue impression had both been scored as plus 1 . Of the 10 biopsies showing Leishmania parasite, 3 showed an RPI of 1 (1-10 organisms per standard) and 7 showed an RPI of 2 (11-100 organisms per standard section).

\section{Discussion}

Material from 78 patients with suspected CL was evaluated. The diagnosis was confirmed as CL, based on the positivity for amastigotes in at least two of the diagnostic methods used in $64.1 \%(50 / 78)$ of biopsies. When numerous amastigotes are present in a biopsy, cutaneous leishmaniasis is easy to diagnose. However, in the latter stages of the cutaneous infection when granulomas predominate and parasite-filled his- tiocytes gradually disappear, it becomes more difficult. Occasionally, the histopathological analysis fails to detect amastigotes even in early lesions [20]. In the current study too, the six biopsies in which organisms were not detected on histology were classified as early lesions of less than 24-week duration. The numbers of late lesions were small, and therefore, although all seven showed organisms on histological examination, this was not statistically significant.

In this study, the 12 cases were excluded since organisms were present in only one of the diagnostic methods used. All 12 cases showed a low parasitic load. CL is usually diagnosed on material obtained from patients with clinically suspicious lesions. Many methods including serology, slit skin smear, culture, histology, and polymerase chain reaction (PCR) are used in the diagnosis of cutaneous leishmaniasis [21]. Microscopic identification of the parasite has superior sensitivity to that of culture [21]. In difficult cases with occasional organisms, the availability of a reliable tool to identify the parasite would be extremely useful. While the inclusion of molecular techniques such as PCR has greatly improved the sensitivity of diagnosis, they are costly and not readily available. Immunohistochemistry is emerging as a new tool in the identification of the Leishmania organism, with studies showing that Cdla immunostaining is useful in the detection of Leishmania organisms [22-25]. This has not been assessed in L. donovani species and may prove to be a useful adjunctive test in equivocal cases and cases of visceral leishmaniasis.

Morphological analysis of the 50 lesions with confirmed CL showed a variable parasite load (discrete to intense) and 
TABLE 2: Dermal changes of lesions among patients with cutaneous leishmaniasis.

\begin{tabular}{|c|c|c|c|}
\hline Dermal change & & Category & Number (\%) \\
\hline \multirow{22}{*}{ Type of inflammatory cells } & \multirow{5}{*}{ Lymphocytes } & Absent & $0(0)$ \\
\hline & & Fewer in number to other cells & $3(6)$ \\
\hline & & Equal in number to other cells & $40(80)$ \\
\hline & & Dominates other cells & $40(80)$ \\
\hline & & Absent & $5(10)$ \\
\hline & \multirow{4}{*}{ Plasma cells } & Fewer in number to other cells & $27(54)$ \\
\hline & & Equal in number to other cells & $18(36)$ \\
\hline & & Dominates other cells & $0(0)$ \\
\hline & & Absent & $0(0)$ \\
\hline & \multirow{3}{*}{ Macrophages } & Fewer in number to other cells & $3(6)$ \\
\hline & & Equal in number to other cells & $25(50)$ \\
\hline & & Dominates other cells & $22(44)$ \\
\hline & \multirow{4}{*}{ Neutrophils } & Absent & $16(32)$ \\
\hline & & Fewer in number to other cells & $32(64)$ \\
\hline & & Equal in number to other cells & $2(4)$ \\
\hline & & Dominates other cells & $0(0)$ \\
\hline & \multirow{6}{*}{ Multinucleated giant cells } & Absent & $43(86)$ \\
\hline & & Fewer in number to other cells & $7(14)$ \\
\hline & & Equal in number to other cells & $0(0)$ \\
\hline & & Dominates other cells & $0(0)$ \\
\hline & & Present & $13(26)$ \\
\hline & & Absent & $37(74)$ \\
\hline \multirow{5}{*}{ Nature of inflammatory infiltrate } & & Macrophage predominant & $22(44)$ \\
\hline & & Lymphocyte predominant & $7(14)$ \\
\hline & & Lymphoplasmacytic & $1(2)$ \\
\hline & & Lymphohistiocytic & $9(18)$ \\
\hline & & Lymphohistioplasmacytic & $11(22)$ \\
\hline \multirow{3}{*}{ Degree of inflammation } & & Mild & $5(10)$ \\
\hline & & Moderate & $16(32)$ \\
\hline & & Extensive & $29(58)$ \\
\hline \multirow{3}{*}{ Necrosis } & & Absent & $40(80)$ \\
\hline & & Focal & $10(20)$ \\
\hline & & Diffuse & $0(0)$ \\
\hline \multirow{2}{*}{ Neuritis } & & Absent & $45(90)$ \\
\hline & & Present & $5(10)$ \\
\hline \multirow{3}{*}{ Granuloma } & & Absent & $9(18)$ \\
\hline & & Ill-defined granuloma & $19(38)$ \\
\hline & & Well defined granuloma & $22(44)$ \\
\hline \multirow{3}{*}{ Pattern of inflammation } & & Diffuse infiltrate & $9(18)$ \\
\hline & & Ill-defined histiocytic infiltrate & $19(38)$ \\
\hline & & Well defined granuloma & $22(44)$ \\
\hline
\end{tabular}

inflammatory infiltrates of variable intensity. Inflammatory infiltrates were characterized by a macrophage predominance, followed by lymphocytes and plasma cells. The epidermis showed both acanthosis and atrophy, accompanied by hyperkeratosis, parakeratosis, spongiosis, exocytosis, and interface changes. Other studies too have demonstrated epidermal changes such as exocytosis in CL [26, 27], highlighting the importance of the epidermis in the immunoregulation of the disease. In addition, lesions demonstrated the presence of ulcers, focal necrosis, and both ill-defined and well-organized granulomas. The presence of wellorganized granulomas with multinucleated giant cells is a 
TABLE 3: Categorization of cases according to Ridley's parasitic index.

\begin{tabular}{lcc}
\hline Score & Parasitic load & No of cases $(\%)$ \\
\hline 6 & More than, 100,000 parasites per standard section & $1(2)$ \\
5 & $10,100-100,000$ parasites per standard section & $0(0)$ \\
4 & $1001-10,000$ parasites per standard section & $9(18)$ \\
3 & $101-1000$ amastigotes per standard section & $10(20)$ \\
2 & $11-100$ amastigotes per standard section & $17(34)$ \\
1 & $1-10$ amastigotes per standard section & $7(14)$ \\
0 & No amastigotes & $6(12)$ \\
& Total & $50(100)$ \\
\hline
\end{tabular}

TABLE 4: Categorization of cases according to modified Ridley index.

\begin{tabular}{|c|c|c|c|}
\hline Group & Morphology & Organisms & No of cases $(\%)$ \\
\hline Group 1 & Parasitized macrophages with variable lymphocytes and plasma cells & + & $8(16)$ \\
\hline Group 2 & $\begin{array}{c}\text { Parasitized macrophages with lymphocytes, plasma cells, and ill- } \\
\text { formed histiocytic granuloma }\end{array}$ & + & $19(38)$ \\
\hline Group 3 & $\begin{array}{c}\text { A mixture of macrophages (with or without parasites), lymphocytes, } \\
\text { plasma cells, and epithelioid granuloma }\end{array}$ & $+/-$ & $17(34)$ \\
\hline Group 4 & $\begin{array}{l}\text { Epithelioid granulomatous response (with or without Langhans type } \\
\text { multinucleated giant cells) with a few lymphocytes and plasma cells } \\
\text { but no amastigotes }\end{array}$ & - & $5(10)$ \\
\hline \multicolumn{3}{|c|}{ Nonspecific chronic inflammation with no amastigotes } & $1(2)$ \\
\hline \multicolumn{3}{|l|}{ Total } & $50(100)$ \\
\hline
\end{tabular}

factor related to the immune system's attempt to eliminate parasites, and the organism load has also been shown to decrease progressively with the development of granulomatous inflammation [12], indicating that the development of granuloma is associated with increased control of the organism. In this study too, well-defined granuloma formation showed a positive association with a low organism load.

The histopathological and immunological host response to the infection caused by parasites of the genus Leishmania depends largely on the infecting parasite [28, 29]. In this study, three main morphological patterns were identified: a diffuse lymphohistioplasmacytic infiltrate, ill-defined histiocytic granuloma, and epithelioid granulomatous inflammation. This is similar to the pattern described by Azadeh et al. in 1985 [11]. The histological spectrum of CL has been classified variably in different studies. Venkataram et al. reported four histological patterns [20]. A more recent study conducted in Panama described three main histopathological patterns: a lymphohistiocytic inflammatory response, a lymphoplasmacytic response, and a granulomatous response [27].

Kurban et al. and Mansour et al. have suggested two histological patterns in biopsies of patients with CL: the first one corresponding to lesions of less than one-year duration associated with a diffuse infiltrate, and the second one corresponding to lesions of more than one-year duration associated with granulomatous infiltrate $[30,31]$. In our study, however, we were not able to demonstrate a statistically significant relationship between the duration of the lesion and morphological pattern, with both early and late lesions demonstrating all three patterns of inflammation Welldefined granulomas were seen in 22 of the lesions, which included both early and late lesions. Well-defined granulomas were seen in a lesion of 660 weeks and a lesion of 4 weeks. Similarly, a diffuse infiltrate was seen in a late lesion of 78 weeks as well as lesions of 4 weeks. Although we were not able to demonstrate a correlation between the duration of the lesion and granuloma formation, well-defined granuloma showed a negative association with the parasite load, indicating that it was the immune response that has a greater correlation with the organism load than the duration of the lesion.

Activation of intact macrophages and a necrotizing process that destroys the macrophage with the parasite it contains are the two main methods of parasite elimination. In both the current study and the previous study conducted in Sri Lanka, necrosis was not a prominent feature and was seen focally in a minority of cases. However, the macrophage was the predominant cell in most cases. This is supportive of the theory that the main mode of parasite elimination in $L$. donovani infection is macrophage activation giving rise to epithelioid granulomata rather than necrosis [12]. Studies have shown T cell mediated IFN- $\gamma$ and TNF- $\alpha$ activate parasite-infected macrophages for parasite destruction [32]. Studies in Sri Lanka showed that the mean percentage of T cells increased with the formation of granuloma and a reduction in parasite counts [12]. Additionally, a Sri Lankan study in which skin biopsies were analyzed for in situ cytokine expression of $\mathrm{T}$ helper cell cytokines showed a prominent $\mathrm{T}$ 
helper 1 response with increased IFN- $\gamma$ levels in healing lesions [33], supporting the hypothesis that macrophage activation plays a key role in parasite elimination in Sri Lanka since IFN- $\gamma$ is a potent activator of macrophages.

At present, there is no vaccine for the use against leishmaniasis and therapeutic options are limited due to factors such as drug toxicity, long treatment courses, challenging routes of drug administration, and instability of drugs in hot climates [34]. An understanding of the immune mechanisms required for the control of infection within the varied tissue microenvironments that contain Leishmania is a key factor for developing effective vaccines and therapeutics. Evaluation of the histopathological features indicates that macrophage activation appears to play a major role. Further characterization of the host immune response in a Sri Lankan population would facilitate a better understanding of hostparasite interaction and mechanisms of effective elimination of the parasite by the host.

\section{Conclusions}

In conclusion, the histopathological changes observed in localized CL caused by L. donovani in Sri Lanka were characterized by a variably intense inflammatory infiltrate with three main histological patterns: a diffuse lymphohistioplasmacytic infiltrate, ill-defined granuloma, and epithelioid granulomatous inflammation. The macrophage was the predominant cell in most cases. Necrosis was not a prominent feature. Organisms were present even in some late lesions of over 24-week duration, and the presence of granuloma did not show a relationship with the duration of the lesion. However, well-formed granuloma formation was associated with a reduction in organism load, indicating that the macrophage activation played an important role in the control of the organism.

\section{Data Availability}

Data can be made available on request from the corresponding author at harshima@path.cmb.ac.lk.

\section{Conflicts of Interest}

The authors declare that there is no conflict of interest regarding the publication of this paper.

\section{Acknowledgments}

Authors would like to acknowledge the National Research Council (NRC), Sri Lanka (Grant No. NRC 16-142) for sharing consumables for the present research activity.

\section{References}

[1] WHO, Leishmaniasis. Key facts, $14^{\text {th }}$ March 2018December 2018, https://www.who.int/news-room/fact-sheets/detail/ leishmaniasis.

[2] J. Alvar, I. D. Vélez, C. Bern et al., "Leishmaniasis worldwide and global estimates of its incidence," PLoS One, vol. 7, no. 5, article e35671, 2012.
[3] WHO, The World Health Report 2004 - Changing history, World Health Organization, Geneva, 2004.

[4] WHO, Control of the leishmaniases, WHO Technical report series 949, World Health Organization, Geneva, 2010.

[5] WHO, WHO Report on Global Surveillance of Epidemic-prone Infectious Diseases - Leishmaniasis, World Health Organization, 2013.

[6] D. Bora, "Epidemiology of visceral leishmaniasis in India," The National Medical Journal of India, vol. 12, no. 2, pp. 62-68, 1999.

[7] D. N. Athukorale, J. K. Seneviratne, R. L. Ihalamulla, and U. N. Premaratne, "Locally acquired cutaneous leishmaniasis in Sri Lanka," The Journal of Tropical Medicine and Hygiene, vol. 95, no. 6, pp. 432-433, 1992.

[8] N. D. Karunaweera, F. Pratlong, H. V. Y. D. Siriwardane, R. L. Ihalamulla, and J. P. Dedet, "Sri Lankan cutaneous leishmaniasis is caused by Leishmania donovani zymodeme MON-37," Transactions of the Royal Society of Tropical Medicine and Hygiene, vol. 97, no. 4, pp. 380-381, 2003.

[9] S. Ranasinghe, W. W. Zhang, R. Wickremasinghe et al., "Leishmania donovani zymodeme MON-37 isolated from an autochthonous visceral leishmaniasis patient in Sri Lanka," Pathogens and Global Health, vol. 106, no. 7, pp. 421-424, 2013.

[10] Y. Siriwardana, G. Zhou, B. Deepachandi et al., "Trends in recently emerged Leishmania donovani induced cutaneous leishmaniasis, Sri Lanka, for the first 13 years," BioMed Research International., vol. 2019, article 4093603, 11 pages, 2019.

[11] B. Azadeh, A. Samad, and S. Ardehali, "Histological spectrum of cutaneous leishmaniasis due to Leishmania tropica," Transactions of the Royal Society of Tropical Medicine and Hygiene, vol. 79, no. 5, pp. 631-636, 1985.

[12] C. Herath, N. Ratnatunga, R. Waduge, P. Ratnayake, C. Ratnatunga, and S. Ramadasa, "A histopathological study of cutaneous leishmaniasis in Sri Lanka," The Ceylon Medical Journal, vol. 55, no. 4, pp. 106-111, 2010.

[13] D. S. Ridley, "The pathogenesis of cutaneous leishmaniasis," Transactions of the Royal Society of Tropical Medicine and Hygiene, vol. 73, no. 2, pp. 150-160, 1979.

[14] D. S. Ridley, "A histological classification of cutaneous leishmaniasis and its geographical expression," Transactions of the Royal Society of Tropical Medicine and Hygiene, vol. 74, no. 4, pp. 515-521, 1980.

[15] D. S. Ridley and M. J. Ridley, "The evolution of the lesion in cutaneous leishmaniasis," Journal of Pathology, vol. 141, no. 1, pp. 83-96, 1983.

[16] D. S. Ridley, W. Peters, and R. Killick-Kendrick, Eds., The Leishmaniases in Biology and Medicine. V.2, Academic Press, London, 1987.

[17] A. Gaafar, A. Y. El Kadaro, T. G. Theander et al., "The pathology of cutaneous leishmaniasis due to Leishmania major in Sudan," American Journal of the Tropical Medicine and Hygiene, vol. 52, no. 5, pp. 438-442, 1995.

[18] A. Ribeiro-de-Jesus, R. P. Almeida, H. Lessa, O. Bacellar, and E. M. Carvalho, "Cytokine profile and pathology in human leishmaniasis," Brazilian Journal of Medical and Biological Research, vol. 31, no. 1, pp. 143-148, 1998.

[19] L. R. V. Antonelli, W. O. Dutra, R. P. Almeida, O. Bacellar, and K. J. Gollob, "Antigen specific correlations of cellular immune responses in human leishmaniasis suggests mechanisms for 
immunoregulation," Clinical and Experimental Immunology, vol. 136, no. 2, pp. 341-348, 2004.

[20] M. Venkataram, M. Moosa, and L. Devi, "Histopathological spectrum in cutaneous leishmaniasis: a study in Oman," Indian Journal of Dermatology, Venereology and Leprology, vol. 67, pp. 294-298, 2001.

[21] H. J. de Vries, S. H. Reedijk, and H. D. Schallig, "Cutaneous leishmaniasis: recent developments in diagnosis and management," American Journal of Clinical Dermatology, vol. 16, no. 2, pp. 99-109, 2015.

[22] A. Fernandez-Flores and J. L. Rodriguez-Peralto, "Morphological and immunohistochemical clues for the diagnosis of cutaneous leishmaniasis and the interpretation of CD1a status," Journal of the American Academy of Dermatology, vol. 74, no. 3, pp. 536-543, 2016.

[23] A. Fernandez-Flores, "A new scenario in the immunohistochemical diagnosis of cutaneous leishmaniasis," Journal of Cutaneous Pathology, vol. 44, no. 12, pp. 1051-1052, 2017.

[24] M. C. Ferrufino-Schmidt, F. Bravo, B. M. Valencia, A. LlanosCuentas, A. K. Boggild, and P. E. LeBoit, "Is CDla useful for leishmaniasis diagnosis in the New World?," Journal of Cutaneous Pathology, vol. 46, no. 1, pp. 90-92, 2019.

[25] L. Sundharkrishnan and J. P. North, "Histopathologic features of cutaneous leishmaniasis and use of CDla staining for amastigotes in Old World and New World leishmaniasis," Journal of Cutaneous Pathology, vol. 44, no. 12, pp. 1005-1011, 2017.

[26] D. M. Isaza, M. Restrepo, R. Restrepo, G. Caceres-Dittmar, and F. J. Tapia, "Immunocytochemical and histopathologic characterization of lesions from patients with localized cutaneous leishmaniasis caused by Leishmania panamensis," The American Journal of Tropical Medicine and Hygiene, vol. 55, no. 4, pp. 365-369, 1996.

[27] K. González, R. Diaz, A. F. Ferreira et al., "Histopathological characteristics of cutaneous lesions caused by Leishmania Viannia panamensis in Panama," Revista do Instituto de Medicina Tropical de São Paulo, vol. 60, article e8, 2018.

[28] D. R. Mehregan, A. H. Mehregan, and D. A. Mehregan, "Histologic diagnosis of cutaneous leishmaniasis," Clinics in Dermatology, vol. 17, no. 3, pp. 297-304, 1999.

[29] F. T. Silveira, R. Lainson, and C. E. P. Corbett, "Clinical and immunopathological spectrum of American cutaneous leishmaniasis with special reference to the disease in Amazonian Brazil: a review," Memórias do Instituto Oswaldo Cruz, vol. 99, no. 3, pp. 239-251, 2004.

[30] A. K. Kurban, J. A. Malak, F. S. Farah, and H. T. Chaglassian, "Histopathology of cutaneous leishmaniasis," Archives of Dermatology, vol. 93, no. 4, pp. 396-401, 1966.

[31] L. Mansour, S. M. el-Marhoumy, M. M. Eid, and K. Gawish, "A histopathological study of different clinical forms of cutaneous leishmaniasis," Journal of the Egyptian Society of Parasitology, vol. 23, no. 2, pp. 591-597, 1993.

[32] G. Cáceres-Dittmar, F. J. Tapia, M. A. Sanchez et al., "Determination of the cytokine profile in American cutaneous leishmaniasis using the polymerase chain reaction," Clinical and Experimental Immunology, vol. 91, no. 3, pp. 500-505, 1993.
[33] N. H. Manamperi, S. Oghumu, N. Pathirana et al., "In situ immunopathological changes in cutaneous leishmaniasis due to Leishmania donovani," Parasite Immunology, vol. 39, article e12413, no. 3, 2017.

[34] G. M. P. Sirimanna, F. K. K. Seneviratne, E. S. N. Samaraweera, R. R. Ranawaka, and C. S. Hulangamuwas, "Guidelines for the management leishmaniasis; Sri Lanka College of Dermatologists Guidelines Formulation Committee," Sri Lanka Journal of Dermatology, vol. 16, pp. 35-45, 2012-2014. 